\title{
Prediabetes and serum insulin levels
}

\author{
Alwaleed Altemani $^{1 *}$, Ali Alamri ${ }^{1}$, Mahir Ahmed ${ }^{2}$, Mosaed Al Garbo ${ }^{3}$, Tariq Alharbi ${ }^{3}$, \\ Rana Al-Rasheed ${ }^{4}$, Saud Alshagraawi ${ }^{5}$, Ibrahim Alzilai ${ }^{6}$, Fuad Alotaibi ${ }^{7}$, Ahlam Al-shahrani ${ }^{8}$
}

\author{
College of Medicine, ${ }^{1}$ King Abdulaziz University, Jeddah, ${ }^{3}$ Almaarefa Colleges for Science \& Technology, Riyadh, \\ ${ }^{5}$ Prince Sattam bin Abdulaziz University, Al-Kharj, Saudi Arabia \\ ${ }^{2}$ Department of Health Control, King Abdulaziz Airport, Jeddah, Saudi Arabia \\ ${ }^{4}$ King Fahd Medical Research Center, King Abdulaziz University, Jeddah, Saudi Arabia \\ ${ }^{6}$ Department of Medicine, South Qunfudah General Hospital, Qunfudah, Saudi Arabia \\ ${ }^{7}$ Saudi Red Crescent Authority, Riyadh, Saudi Arabia \\ ${ }^{8}$ College of Medicine, King Khalid University, Abha, Saudi Arabia
}

Received: 24 March 2018

Accepted: 12 April 2018

\section{*Correspondence:}

Dr. Alwaleed Altemani,

E-mail: alwaleed.fahad@hotmail.com

Copyright: ( ) the author(s), publisher and licensee Medip Academy. This is an open-access article distributed under the terms of the Creative Commons Attribution Non-Commercial License, which permits unrestricted non-commercial use, distribution, and reproduction in any medium, provided the original work is properly cited.

\begin{abstract}
Prediabetes remains a diagnostic dilemma. It refers to impaired glycaemic values without reaching the threshold for diagnosing diabetes mellitus. Prediabetes is an important risk factor for the development of diabetes mellitus, and it constitutes the stage during which microvascular and macrovascular complications are initiated. Early and accurate identification of this stage is the gold standard for prevention of diabetes and its consequences. Despite the multiplicity of diagnostic tests proposed for identification of this condition, a reliable test remains elusive. This article aims at reviewing the different available tests for diagnosis of prediabetes states with a focus on serum insulin levels. Insulin plays a major role in the pathophysiology and development of prediabetes. Different mechanisms of insulin resistance and insulin secretion are established in different subtypes of prediabetes. Therefore, fasting insulin level seems to be a reliable and promising tool for diagnosis and management of prediabetes.
\end{abstract}

Keywords: Prediabetes, Insulin level

\section{INTRODUCTION}

Prediabetes is a state of hyperglycaemia characterized by elevation of blood glucose levels higher than normal but lower than the values of frank diabetes. ${ }^{1-3}$ To date, prediabetes remains a topic of concern for many researchers because of its deleterious complications on health. Prediabetes does not only put the patient at higher risk for developing diabetes mellitus, but is also associated with negative consequences on renal, retinal, and peripheral nervous tissues. ${ }^{4,5}$ The benefit of identification of this state is that multiple prospective studies confirmed that early lifestyle modifications and dietary intervention at this prediabetic state had significantly improved the outcome and prevented longterm complications. ${ }^{6}$

Despite the well-established risk and consequences of prediabetes, conflicts remain about the definition, diagnostic criteria, and diagnostic tests of this state. Fasting plasma glucose level, oral glucose tolerance test, and haemoglobin A1c tests are used for the diagnosis of prediabetes, and recent studies suggest insulin level can also be useful for diagnosis.

This article will discuss the definition, epidemiology, diagnosis, and pathophysiology, and it will focus on insulin levels in this state. 


\section{PREDIABETES DEFINITION}

Prediabetes is an intermediate state of hyperglycaemia in which glycaemic measures (such as glucose level and $\mathrm{HbA1c}$ ) are elevated at levels higher than normal but lower than the diagnostic thresholds for diabetes mellitus. ${ }^{2}$ It has longer been accepted that prediabetes is a risk factor for developing diabetes mellitus in the future. However, recent researchers argued the importance of not defining prediabetes on a glucocentric basis because it is well-established that this state of hyperglycaemia is associated with microvascular dysfunction, macrovascular consequences and early end-organ damage same as diabetes. Retinopathy, neuropathy, and nephropathy were found in up to $10 \%$ of prediabetic patients. Similarly, prediabetes was also associated with metabolic syndrome, cognitive disturbances, neoplasms, hormonal changes, fatty liver disease, and obstructive sleep apnoea. ${ }^{4}$

Currently, there are many definitions for prediabetes that are mainly based on biochemical measures. Each organization propose a different definition for prediabetes depending on its sensitivity and specificity to identify the individuals at risk for developing microvascular and macrovascular complications of the poor glycaemic control, and the morbidity and mortality associated. ${ }^{2}$ The American Diabetes Association (ADA), the World Health Organization (WHO), and the International Expert Committee define prediabetes depending on four main markers namely fasting blood glucose, oral glucose tolerance test, combined fasting blood glucose and glucose tolerance test, and haemoglobin A1c levels. ${ }^{3}$

Because none of the aforementioned glycaemic measures were accurate and reliable for diagnosing and predicting the outcome of prediabetes, new multidimensional approaches are suggested for defining prediabetes. These approaches propose adding other factors (e.g. the existence of microvascular or macrovascular complications of diabetes) to the cut-off thresholds for glycaemic variables. ${ }^{2}$ Such definition would better identify individuals at risk at earlier stages of the disease. However, this idea is still under research and need extensive discussion and studying of cost-effectiveness. Developing a standardized reliable definition for prediabetes is essential not only to prevent the progression of the state to frank diabetes, but also to delay the development of microvascular or macrovascular complications of impaired glycaemic states.

\section{EPIDEMIOLOGY AND RISK FACTORS FOR PREDIABETES}

Prediabetes is more prevalent than diabetes worldwide ${ }^{4}$, and the figure is expected to increase over the next decades. ${ }^{8}$ The prevalence is variable according to the definition of prediabetic state as well as the ethnic group. For instance, a review study about the prevalence of prediabetes among ethnicities reported that prediabetes was more common among Caucasian. The prevalence of impaired fasting plasma glucose was $58.0 \%$ among Caucasian and $48.1 \%$ among Asian. Impaired glucose tolerance, however, was prevalent among $20.3 \%$ of Caucasian and $27.7 \%$ of Asian. ${ }^{9}$ According to the National Diabetes Statistics reports $(2017)^{10}$, it is estimated that about $44 \%$ of the United States adults and $43 \%$ of elderly above 65 years have prediabetes. Only $11.6 \%$ of them were not educated by their healthcare professionals about their condition. Prediabetes was stated in this report to be more prevalent among men $(36.6 \%)$ than women $(29.3 \%){ }^{10}$

Risk factors for prediabetes have been studies by many researchers. Abdominal obesity, hypertension, and low HDL-cholesterol levels were the most significant modifiable risk factors for prediabetes. ${ }^{11}$ Abdominal obesity was the strongest factor associated with prediabetes in both genders suggesting that it is an independent risk factor for this hyperglycaemic state. Male gender was the most established non-modifiable factor associated with high risk for development of prediabetes. $^{12}$ Sex, body mass index (BMI) and family history of diabetes were predictors for progression to type two diabetes mellitus among prediabetic patients., ${ }^{7,12}$ Thus, screening for prediabetes is recommended by the American Diabetes Association (ADA) 2016 to be performed for all overweight or obese individuals (BMI $>85^{\text {th }}$ percentile for age and sex, weigh $>120 \%$ of ideal weight, or weight for height $>85^{\text {th }}$ percentile) with two or more risk factors including: first or second-degree relative with type 2 diabetes, maternal history of gestational diabetes during the child's pregnancy type diabetes, signs of insulin resistance (e.g. acanthosis nigricans, dyslipidemia, hypertension, polycystic ovarian syndrome), or certain ethnic group such as African American, Native American, Pacific Islander, Latino, or Asian American descent. ${ }^{13}$

Management of prediabetes is primarily nonpharmacological. Lifestyle modifications are the mainstay treatment for individuals with impaired glycaemic measures. Weight loss, regular exercise, health low-fat and high fibre-diet, active non-sedentary life, and smoking cessation are effective measures for lowering blood glucose levels to normal values. Some alternative therapies such as banaba, fenugreek, magnesium, ginseng, white mulberry, gymnema, and cassia cinnamon are proposed, but not yet established as effective measures for treating prediabetes.

\section{DIAGNOSIS OF PREDIABETES}

According to the American diabetic Association (ADA) 2016 clinical practice recommendations, prediabetes can be diagnosed on basis of three tests: fasting blood glucose, oral glucose tolerance test, and HbA1c levels. ${ }^{13}$ Prediabetes is defined as fasting blood glucose between $100-125 \mathrm{mg} / \mathrm{dl}$ (5.6-6.9 mmol/l), Two-hour postprandial glucose level between 140-199 mg/dl (7.8-11.0 mmol/l), 
or haemoglobin A1C level between 5.7-6.4\% (39-46 $\mathrm{mmol} / \mathrm{mol}$ ).

The first consensus-based criteria for definition of prediabetes were proposed by the American National Diabetes Data Group (NDDG) association in 1979 and were changed several times. Basically, prediabetes (or non-diabetic hyperglycaemia) diagnostic criteria included: a fasting plasma glucose concentration less than that defined for the diagnosis of diabetes $(<140 \mathrm{mg} / \mathrm{dl}), 2$ hour oral glucose post plasma level between 140-199 $\mathrm{mg} / \mathrm{dl}$, and a midtest plasma glucose value of $\geq 200 \mathrm{mg} / \mathrm{dl}$. Clinical manifestations are not included in the diagnostic criteria of prediabetes. Therefore, many terms were proposed for defining this non-diabetic hyperglycaemic state e.g. "chemical diabetes", "borderline diabetes", "impaired glucose tolerance", and recently "prediabetes". 14 The World Health Organisation (WHO) is different from the American National Diabetes Data Group (NDDG) in defining prediabetes as regards some points. For instance, the WHO consider a person prediabetic when his fasting plasma glucose and 2-hour post prandial glucose values are elevated without requiring an additional midtest value of $\geq 200 \mathrm{mg} / \mathrm{dl} .{ }^{15}$ This definition is more inclusive than the NDDG group definition because in includes patients with impaired fasting glucose and impaired 2-hour glucose tolerance with midtest glucose levels $<200 \mathrm{mg} / \mathrm{dl} .{ }^{15}$

\section{Fasting plasma glucose}

Fasting plasma glucose (FPG) level is measured after 6-8 hours of fasting. It is considered normal if the level was below $100 \mathrm{mg} / \mathrm{dl}(5.6 \mathrm{mmol} / \mathrm{l})$. Prediabetes is considered when FPG level lies between 100 and $125 \mathrm{mg} / \mathrm{dl}$ (5.6-6.9 $\mathrm{mmol} / \mathrm{l}$ ), and diabetes is diagnosed when the level is 126 $\mathrm{mg} / \mathrm{dl}(7.0 \mathrm{mmol} / \mathrm{l})$ or more. ${ }^{13}$ Cut-off values for impaired fasting plasma glucose (prediabetes) were a subject for discussion. For example, the ADA in 2003 recommended lowering the cut-off value of impaired fasting glucose to $100 \mathrm{mg} / \mathrm{dl}(5.6 \mathrm{mmol} / \mathrm{l})$ based on the results of review studies to maximize the sensitivity of detecting early prediabetes and consequently preventing diabetes. This lowering was associated with lower cardiovascular complications and lower risk for developing diabetes. ${ }^{16}$ However, the WHO refused lowering this cut-off value and maintained the defining value for impaired fasting glucose at $110 \mathrm{mg} / \mathrm{dl}$ to avoid the major increase in prevalence of prediabetes with its social, economic, and personal negative consequences. In fact, an exact value for defining a cut-off level for prediabetes remain a matter of challenge because one of the studies indicated that the mild increase of fasting plasma glucose from 81 $\mathrm{mg} / \mathrm{dl} \quad(4.5 \mathrm{mmol} / \mathrm{l})$ to $86 \mathrm{mg} / \mathrm{dl}(4.8 \mathrm{mmol} / \mathrm{l})$ was significantly associated with increased risk for developing type 2 diabetes mellitus. ${ }^{16}$ Similarly, $50 \%$ of patients with fasting plasma glucose $\geq 106 \mathrm{mg} / \mathrm{dl}$ (5.9 $\mathrm{mmol} / \mathrm{l}$ ) developed diabetes in another study. ${ }^{17}$ This indicates that the reliability of using fasting plasma glucose as an indicator for prediabetes diagnosis remains debatable. Therefore, the diagnostic criteria for prediabetes were subjected to major changes over time.

\section{Oral glucose tolerance test}

Oral glucose tolerance test is performed through giving the patient $75 \mathrm{~g}$ of glucose after an eight-hour period of fasting, then measuring the post prandial plasma glucose level after 2 hours. A 2-hour postprandial plasma level less than $140 \mathrm{mg} / \mathrm{dl}(7.8 \mathrm{mmol} / \mathrm{l})$ is normal. Levels between 140 and $199 \mathrm{mg} / \mathrm{dl}(7.8-11.0 \mathrm{mmol} / \mathrm{l})$ are definitive for prediabetes, and levels $\geq 200 \mathrm{mg} / \mathrm{dl}(11.1$ $\mathrm{mmol} / \mathrm{l}$ ) are diagnostic for diabetes mellitus. ${ }^{13}$ The reason behind inclusion of impaired glucose tolerance (IGT) as a criterion for diagnosis of prediabetes is that it carries a considerably higher risk for developing type 2 diabetes mellitus in comparison to individuals with normal glucose tolerance. About 1-5\% of patients with IGT develop diabetes yearly, whilst less than $1 \%$ of individuals with normal glucose tolerance do. On the other hand, up to $10 \%$ of patients with IGT remains in the IGT state for 10 years without developing diabetes. IGT is also not essentially an independent risk factor for the development of nephropathy, neuropathy, or retinopathy. However, it was found to be associated with higher mortality rates due to cardiovascular diseases. ${ }^{1}$

\section{Glycated haemoglobin HbAlc levels}

Haemoglobin A1C (HbA1C) use was introduced later on and was established as an important criterion for defining prediabetes. HbA1C indicated the average plasma glucose level for the past 2-3 months. It measures the percentage of glycated-haemoglobin that reflects the hyperglycaemic state during the half-life of the red blood cells tested. In general, HbA1C level below $5.7 \%$ is considered normal, prediabetes is defined with $\mathrm{HbA} 1 \mathrm{C}$ level between 5.7 and $6.4 \%$, and diabetes is diagnosed when $\mathrm{HbA1C}$ is $\geq 6.5 \%$ on two separate tests. ${ }^{18}$ Haemoglobin A1C reflects long-term glucose concentration rather than a point measure that can be affected by food intake. It is also a better representative indicator for chronic complications of impaired glycaemic control. In spite of its higher cost, its use is a rationale approach for diagnosis for diagnosis of prediabetes. Whereas a value of $6.5 \%$ is the cut-off value for diabetes, a level of $6 \%$ was initially proposed for diagnosing prediabetes. Individuals with HbAlc levels between 6 and $6.5 \%$ were 10 times riskier for developing diabetes than those with $\mathrm{HbA1C}$ levels $\leq 6 \%$. However, debates also exists about this cut-off value for defining prediabetes because many studies had reported high risk of diabetes among individuals with lower $\mathrm{HbA1C}$ levels $(5.5 \%$ and $5.7 \%) .{ }^{19}$ Therefore, the latest ADA diagnostic criteria adopted the value of $5.7 \%$ as a cut-off level for diagnosing prediabetes. ${ }^{13}$ However, further studies are recommended for identifying a reliable level for predicting the actual risk for developing diabetes mellitus. 


\section{INSULIN AND PREDIABETES}

As aforementioned, many diagnostic tests have been proposed and established for diagnosis of prediabetes. And despite their multiplicity, a reliable test remains elusive. Serum insulin level is a promising test for diagnosing prediabetes and predicting the risk of developing diabetes mellitus. Various epidemiological studies reported that the pathophysiological characters of impaired fasting plasma and impaired glucose tolerance were significantly different from the actual pathophysiology of diabetes. $^{20}$ Low insulin level and insulin resistances are the main pathophysiological mechanisms in type 2 diabetes mellitus. Therefore, recent researches are concerned with studying the role of measuring insulin levels as well as insulin resistance as predictive indicators for diabetes in prediabetic hyperglycemic states. In type 2 diabetes, there is either low insulin level or considerable insulin resistance in spite of high insulin level in the blood. ${ }^{16}$ Insulin resistance refer to the low response of different tissues and organs to respond to endogenous or exogenous insulin leading to increased plasma glucose levels. ${ }^{21}$

\section{Insulin resistance as a pathophysiology of prediabetes}

In prediabetes, it has been found that insulin resistance is significantly higher than individuals with normal glucose levels. ${ }^{16}$ Of interest, there are different mechanisms for insulin resistance among prediabetic patients in relation to the glycaemia-measuring tools used. For instance, patients with isolated impaired fasting plasma glucose display a considerable hepatic insulin resistance, whilst patients with isolated impaired glucose tolerance exhibit significant peripheral insulin resistance. ${ }^{22,23}$ It is stated that the main pathophysiologic mechanism in prediabetic patients with impaired fasting plasma glucose is impaired suppression of hepatic production of glucose in spite of hyperinsulinemia. Hepatocytes continue to synthetize excessive amount of glucose and seem to be nonresponsive to elevated blood insulin levels. In contrast, peripheral insulin resistance remains intact with values closely similar to the normal euglycemic individuals. Thus, hepatic insulin resistance is proposed to be the main pathophysiological mechanism in impaired fasting glucose state. ${ }^{22}$ Conversely, prediabetics with impaired glucose tolerance have significantly higher peripheral insulin resistance, when measured with the euglycemic hyperinsulinemic clamp, when compared to normal individuals. $^{24}$ Prediabetics who have both impaired glucose tolerance and impaired fasting glucose exhibit both peripheral and hepatic resistance. ${ }^{22}$

\section{Insulin secretion in prediabetes}

In its essence, diabetes develops due to the progressive failure of the pancreatic beta cells ti produce insulin. Insulin secretion is stimulated by glucose administration either orally or parenterally. Oral administration promotes insulin secretion not only via direct stimulation of the pancreas but also through the additional effect of incretin, a metabolic hormone that is secreted after eating. After glucose administration, insulin is secreted from the pancreas in two phases: an early phase characterized by a sharp increase in insulin level and a late slower phase of secretion. $^{25}$

In prediabetes, failure of pancreatic beta cells to secrete adequate amounts of insulin is the earliest pathophysiological mechanism of hyperglycemia. Pancreatic beta cell failure mechanisms are different according to the subtype of prediabetes. In isolated impaired glucose tolerance subtype, early phase insulin secretion is significantly lower than normal individuals, whilst late stage phase secretion is closely similar to healthy population. Other studies reported reduction of both early and late phase insulin levels among those patients. ${ }^{23}$ In contrast, prediabetics with isolated impaired fasting glucose have significantly lower late-phase insulin secretion and relatively intact early-phase insulin levels in comparison with normoglycemic controls. ${ }^{26,27}$ Furthermore, the two subtypes of prediabetes are different as regards the stimulatory mechanisms for insulin secretion. In prediabetics with isolated fasting glucose impairment, disorder of insulin secretion results from direct pancreatic beta cell dysfunction after oral and intravenous glucose administration, whereas incretin dysfunction plays a role when impaired glucose tolerance is encountered. ${ }^{24,27}$

Hence, there seems to be a considerable difference in the pathophysiological mechanisms of the two subtypes of prediabetes. Considering these different mechanisms can be of a clinical significance when thinking about the management of prediabetes. Basically, the mainstay management of prediabetes was non-pharmacological. It focused on lifestyle changes, and dietary modifications, and weight loss. These simple non-pharmacological measures were proved to improve the glycemic control, prevent the development of frank diabetes, and delay the development of microvascular complications. ${ }^{28}$ However, when pharmacological interventions are to studied according to the proposed insulin resistance and insulin secretion mechanisms, more positive outcomes may be encountered. Incretin-based therapies and GLP-1 antagonists are currently studied in prediabetes with promising results. However, the safety of these agents remains a topic of debate. ${ }^{29,30}$

\section{Insulin level for diagnosis of prediabetes}

Given the important pathophysiologic role of insulin in prediabetes, many researchers argue the importance of measuring insulin level as reliable criterion for diagnosis of prediabetes. Johnson et al, in their retrospective study on 965 diabetic patients, reported that fasting insulin level of $9.0 \mathrm{micro} \mathrm{IU} / \mathrm{ml}$ could found in $80 \%$ of patients during the prediabetes states, indicating that it may be considered the most reliable clinical tool for identifying prediabetes. ${ }^{31}$ Similarly, a more recent study reported that 
using insulin metabolites to measure insulin resistance was another reliable test for diagnosis of prediabetes. ${ }^{32}$ Despite the few reported literature study about insulin level measurement in prediabetes, it seems to be a promising reliable tool for accurate diagnosis of this state.

\section{CONCLUSION}

Prediabetes is an important risk factor for the development of diabetes mellitus, and it constitutes the stage during which microvascular and macrovascular complications are initiated. Early and accurate identification of this stage is the gold standard for prevention of diabetes and its consequences. Despite the multiplicity of diagnostic tests proposed for identification of this condition, a reliable test remains elusive. Insulin plays a major role in the pathophysiology and development of prediabetes. Different mechanisms of insulin resistance and insulin secretion are established in different subtypes of prediabetes. Therefore, fasting insulin level seems to be a reliable and promising tool for diagnosis and management of prediabetes.

Funding: No funding sources

Conflict of interest: None declared

Ethical approval: Not required

\section{REFERENCES}

1. Goldenberg R, Punthakee Z. Definition, Classification and Diagnosis of Diabetes, Prediabetes and Metabolic Syndrome. Can J Diabetes. 2013;37(1):8-11.

2. Vas PRJ, Alberti KG, Edmonds ME. Prediabetes: moving away from a glucocentric definition. Lancet Diabetes Endocrinol. 2017;5(11):848-9.

3. Færch K, Witte DR, Brunner EJ, Kivimäki M, Tabák A, Jørgensen ME, et al. Physical Activity and Improvement of Glycemia in Prediabetes by Different Diagnostic Criteria. The Journal of clinical endocrinology and metabolism. 2017;102(10):371221.

4. Tabák AG, Herder C, Rathmann W, Brunner EJ, Kivimäki M. Prediabetes: A high-risk state for diabetes development. Lancet. 2012;379(9833):2279-90.

5. Souza CF, Gross JL, Gerchman F, Leitao CB. Prediabetes: diagnosis, evaluation of chronic complications, and treatment. Arq Bras Endocrinol Metab. 2012;56(5):275-84.

6. Bansal N. Prediabetes diagnosis and treatment: A review. World J Diabetes. 2015;6(2):296.

7. Watson CS. Prediabetes: Screening, Diagnosis, and Intervention. J Nurse Pract. 2017;13(3):216-221.e1.

8. Abraham TM, Fox CS. Implications of rising prediabetes prevalence. Diabetes Care. 2013;36(8):2139-41.

9. Yip WCY, Sequeira IR, Plank LD, Poppitt SD. Prevalence of pre-diabetes across ethnicities: A review of impaired fasting glucose (IFG) and impaired glucose tolerance (IGT) for classification of dysglycaemia. Nutrients. 2017;9(11):1273.

10. Centers for Disease Control and Prevention. National Diabetes Statistics Report; 2017; 2017.

11. Díaz-Redondo A, Giráldez-García C, Carrillo L, Serrano R, García-Soidán FJ, Artola $\mathrm{S}$, et al. Modifiable risk factors associated with prediabetes in men and women: A cross-sectional analysis of the cohort study in primary health care on the evolution of patients with prediabetes. BMC Fam Pract. 2015;16(1):5.

12. Kawada T. Risk factors for developing prediabetes. Diabetes Res Clin Pract. 2018;135:232.

13. American Diabetes Association. 2016 American Diabetes Association (ADA) Diabetes Guidelines Summary Recommendation from NDEI. Natl Diabetes Educ Initiat. 2016;39(1):1-46.

14. Retnakaran R, Qi Y, Sermer M, Connelly PW, Zinman B, Hanley AJG. Comparison of National Diabetes Data Group and American Diabetes Association diagnostic criteria for gestational diabetes in their identification of postpartum risk of glucose intolerance. Diabetes Res Clin Pract. 2009;85(1):40-6.

15. Seino $\mathrm{Y}$, Nanjo $\mathrm{K}$, Tajim $\mathrm{N}$, Kadowaki $\mathrm{T}$, Kashiwagi A, Araki E, et al. Report of the committee on the classification and diagnostic criteria of diabetes mellitus. J Diabetes Investig. 2010;1(5):212-28.

16. Rhee SY, Woo JT. The prediabetic period: Review of clinical aspects. Diabetes Metab J. 2011;35(2):107-16.

17. The ARIC Investigators. The Atherosclerosis Risk in Communities (ARIC) Study: design and objectives. The ARIC investigators. Am J Epidemiol. 1989;129(4):687-702.

18. Kilpatrick ES, Bloomgarden ZT, Zimmet PZ. Is haemoglobin A1c a step forward for diagnosing diabetes? BMJ. 2009;339:4432.

19. Olson DE, Rhee MK, Herrick K, Ziemer DC, Twombly JG, Phillips LS. Screening for diabetes and pre-diabetes with proposed A1C-based diagnostic criteria. Diabetes Care. 2010;33(10):2184-9.

20. Unwin N, Shaw J, Zimmet P, Alberti KGMM. Impaired glucose tolerance and impaired fasting glycaemia: The current status on definition and intervention. Diabetic Medicine. 2002;19:708-23.

21. Lebovitz HE. Insulin resistance: Definition and consequences. Exp Clin Endocrinol Diabetes. 2001;109(2).

22. Ferrannini E, Gastaldelli A, Iozzo P. Pathophysiology of Prediabetes. Med Clin North Am. 2011;95(2):327-39.

23. Abdul-Ghani MA, Jenkinson CP, Richardson DK, Tripathy D, DeFronzo RA. Insulin secretion and action in subjects with impaired fasting glucose and impaired glucose tolerance: Results from the 
veterans administration genetic epidemiology study. Diabetes. 2006;55(5):1430-5.

24. Bock G, Man CD, Campioni M, Chittilapilly E, Basu R, Toffolo G, et al. Pathogenesis of prediabetes: Mechanisms of fasting and postprandial hyperglycemia in people with impaired fasting glucose and/or impaired glucose tolerance. Diabetes. 2006;55(12):3536-49.

25. Færch K, Vaag A, Holst JJ, Glümer C, Pedersen O, Borch-Johnsen K. Impaired fasting glycaemia vs impaired glucose tolerance: Similar impairment of pancreatic alpha and beta cell function but differential roles of incretin hormones and insulin action. Diabetologia. 2008;51(5):853-61.

26. Kærch K, Vaag A, Holst JJ, Hansen T, Jøhnsen T, Borch-Johnsen K. Natural history of insulin sensitivity and insulin secretion in the progression from normal glucose tolerance to impaired fasting glycemia and impaired glucose tolerance: The inter99 study. Diabetes Care. 2009;32(3):439-44.

27. Rhee SY, Kim JY, Chon S, Hwang YC, Jeong IK, $\mathrm{Oh} \mathrm{S}$, et al. The changes in early phase insulin secretion in newly diagnosed, drug naive korean prediabetes subjects. Korean Diabetes J. 2010;34(3):157-65.
28. Tuomilehto J, Indstrom J, Eriksson J, Valle T, HE\& UM. Prevention of Type 2 Diabetes Mellitus By Changes in Lifestyle Among Subjects With Impaired Glucose Tolerance. $\mathrm{N}$ Engl $\mathrm{J}$ Med. 2001;344(18):1343-50.

29. Papaetis GS. Incretin-based therapies in prediabetes: Current evidence and future perspectives. World J Diabetes. 2014;5(6):817.

30. Prasad-Reddy L, Isaacs D. A clinical review of GLP-1 receptor agonists: Efficacy and safety in diabetes and beyond. Drugs Context. 2015;4.

31. Johnson J, Duick D, Chui M, Aldasouqi S. Identifying Prediabetes Using Fasting Insulin Levels. Endocr Pract. 2010;16(1):47-52.

32. Cobb J, Gall W, Adam KP, Nakhle P, Button E, Hathorn J, et al. A novel fasting blood test for insulin resistance and prediabetes. J Diabetes Sci Technol. 2013;7(1):100-10.

Cite this article as: Altemani A, Alamri A, Ahmed M, Al Garbo M, Alharbi T, Al-Rasheed R, et al. Prediabetes and serum insulin levels. Int $\mathrm{J}$ Community Med Public Health 2018;5:1684-9. 